\title{
RADIATION THERAPY-INDUCED SUBACUTE INTESTINAL OBSTRUCTION
}

\author{
RUPAM G' ${ }^{1}$, BALAJI $0^{1}$, SEREEN RT ${ }^{1}$, NAVIN PATIL ${ }^{2 *}$
}

${ }^{1}$ Department of Pharmacology, Kasturba Medical College, Manipal University, Manipal, Karnataka, India. ${ }^{2}$ Department of Pharmacology, Kasturba Medical College, Manipal University, Manipal, Karnataka, India. Email: navin903@gmail.com

Received: 16 March 2017, Revised and Accepted: 19 April 2017

ABSTRACT

Radiation therapy is an important treatment modality for malignancies of the cervix, uterus, prostate, bladder, testes, and rectum. The incidence of clinically significant radiation-related complications is about 5-20\%. Radiation insult to gastrointestinal tract includes damage to the small bowel, colon, and rectum. Predisposing risk factors such as previous surgery, hypertension, diabetes, smoking, hyperbaric oxygen increases the severity of damage as well as the use of other chemotherapeutic agents increases the sensitivity to radiation. Radiation-induced injury can be acute or chronic depending on the mitotic activity of intestinal tissue. Radiation enteritis is usually managed conservatively at the start, but surgery is indicated when complications arise. Hereby, we report a case of subacute intestinal obstruction after 3 weeks of radiotherapy.

Keywords: Intestinal obstruction, Radiotherapy, Radiation enteritis, Adverse reaction.

(C) 2017 The Authors. Published by Innovare Academic Sciences Pvt Ltd. This is an open access article under the CC BY license (http://creativecommons. org/licenses/by/4. 0/) DOI: http://dx.doi.org/10.22159/ajpcr.2017.v10i7.18520

\section{INTRODUCTION}

Radiation therapy is an important treatment modality form alignancies of the cervix, uterus, prostate, bladder, testes, and rectum and it constitutes almost $40 \%$ in the treatment of major cancers [1]. The incidence of clinically significant radiation-related complications is about $5-20 \%$. Radiation insult to gastrointestinal tract includes damage to the small bowel, colon, and rectum [2]. Subacute intestinal obstruction being one of the most dreaded side effects among them. Radiation enteritis has been estimated to occur in $2-5 \%$ of patients receiving abdominal or pelvic radiotherapy $[3,4]$. The 10 -year incidence of small bowel injuries is estimated at $8 \%$, and that of severe injuries such as bleeding, intestinal obstruction, stenosis, fistula formation, malabsorption, and peritonitis is estimated at $30 \%$ [4]. Hereby, we report a case of subacute intestinal obstruction after 3 weeks of radiation therapy.

\section{CASE REPORT}

Informed consent was obtained from the patient. A 60-year-old female, a known case of carcinoma cervix Stage IV B, with computed tomography suggestive of para-aortic lymph nodes enlargement was planned for high palliation radiotherapy and received 60 gray units per 30 fractions external beam radiotherapy for a total of 6 weeks. Abdominal X-ray was normal (Fig. 1). The patient tolerated the radiotherapy well with Grade II dermatitis. However, after 6 weeks, she complained of severe fatigue, dizziness, fever with chills, abdominal pain along with two episodes of vomiting, exertional chest pain, and obstipation and additionally reported dysuria and appearance of purulent thick pus in the urine. Thorough examination revealed a temperature $102^{\circ} \mathrm{F}$, blood pressure of $150 / 90 \mathrm{mmHg}$ with sinus tachycardia, low volume pulse, and elevated jugular venous pulse. Abdominal examination affirmed diffuse pain associated with severe rigidity and guarding. X-ray erect abdomen showed few air-filled bowel loops in the right lumbar and epigastric region (Fig. 2). She had no other medical or surgical comorbidities. Thus, a diagnosis of radiotherapy-induced intestinal obstruction along with co-associated with catheter-related urinary sepsis was made, and the patient was started on injection piperacillin-tazobactam combination and local application of gentian violet for dermatitis.

\section{DISCUSSION}

Radiation enteritis has been estimated to occur in $2-5 \%$ of patients receiving abdominal or pelvic radiotherapy $[3,4]$. Radiation damage to the small bowel tissue can present as acute or chronic enteritis. Acute radiation-induced small bowel disease presents with colicky abdominal pain, bloating, loss of appetite, nausea, diarrhea, and fecal urgency during or shortly after the course of radiotherapy. These symptoms appear usually during the $2^{\text {nd }}$ week of treatment, as tissue damage and inflammation is maximum during this time, and they peak by $4^{\text {th }}$ to $5^{\text {th }}$ week, when histological changes are stable [2].

Chronic enteropathy is characterized by post-prandial pain, acute or intermittent small bowel obstruction, nausea, anorexia, weight loss, bloating, diarrhea, steatorrhea and malabsorption of selected or multiple nutrients, fistula formation, urethral stenosis, cystitis, and perforation. These can arise from damage to the small bowel itself or associated phenomena such as bile salt malabsorption, bacterial overgrowth, or lactose intolerance [3].

Intestinal damage and symptom severity are related to the radiation regime, size, and site of treatment field, amount of radiation encountered, area of normal bowel being exposed, use of concurrent chemotherapy and the presence of radiation implants. Symptoms may start at an initial dose of 5-12 gray in a fractionated course, but becomes more severe at higher doses [2].

Other predisposing risk factors increasing the risk of radiation injury are previous surgeries that cause development of adhesions, hypertension, diabetes, smoking, thin built post-menopausal females, inflammatory bowel disease, administration of hyperbaric oxygen at the time of radiation to escalate tumor destruction that makes healthy cells also susceptible, and concomitantly administered chemotherapeutic agents furthermore increases the sensitivity to radiation [2]. These factors may also restrict blood flow to the bowel wall, thereby aggravating radiation injury.

Mechanism of radiation enteritis - gastrointestinal tract is second only to kidneys in radiosensitivity with more predominant involvement of small bowel compared to large bowel. Radiotherapy damages the mucosa more severely relatively to other intestinal layers and the reason usually given is due to rapid cell proliferation causing a rupture in the cell cycle which leads to villous atrophy, acute inflammation, and fibrosis. Due to progressive obliterating vasculitis, ischemia results in vascular thrombosis along with intestinal wall fibrosis and 


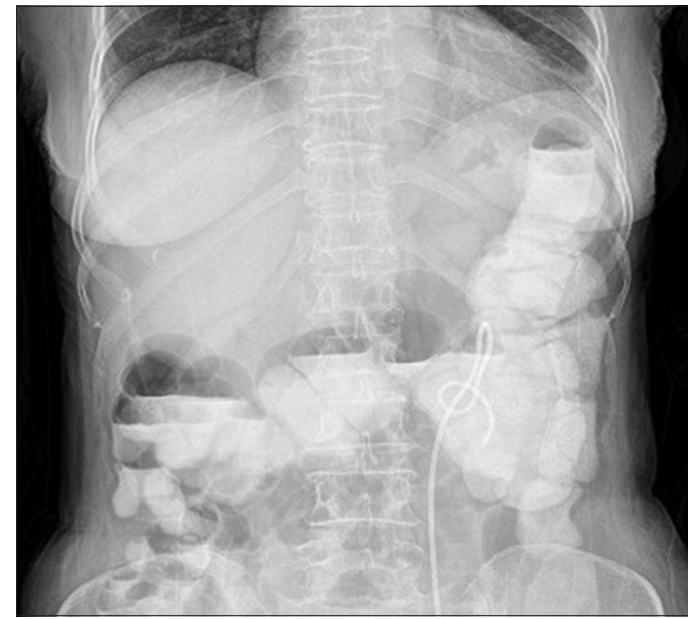

Fig. 1: Normal abdominal X-ray before radiotherapy

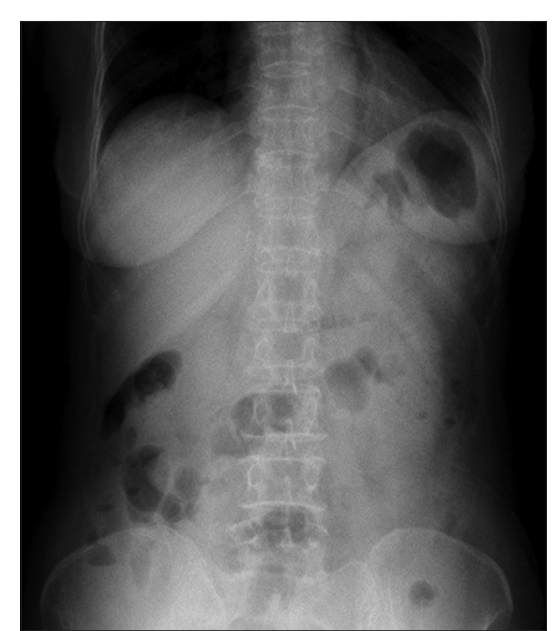

Fig. 2: Abnormal abdominal X-ray showing air-filled bowel loops in the right lumbar and epigastric region

necrosis [5-7]. The radiation-induced injury is best described in two ways - acute injury is caused by injury to the mitotically active intestinal crypt cells; on the other hand, chronic radiation injury is caused by injury to the less mitotically active vascular endothelial and connective tissue cells. Complete histologic recovery takes 6 months. Chronic effects usually manifest after 6-24 months and are caused mostly by obliterative arteritis and thrombosis of vessels; the result is ischemia or necrosis. A reduction in field size, multiple field arrangements, conformal radiotherapy techniques, and intensity-modulated radiotherapy can reduce toxicity related to radiotherapy. Surgical placement of absorbable mesh slings and silicone prosthesis have been described to prevent radiation-induced small bowel disease. Only $20 \%$ of the patients are referred for gastro-enterologic evaluation because symptoms are either underestimated or not recognized [2].

In our case, the only risk factor includes a thin built post-menopausal female. Radiotherapy Oncology Group of Philadelphia has proposed a way to stage lesion grade and based on it this case falls in Grade I acute radiation morbidity [8]. It was suspected to be obstruction and patient was kept nil per oral and was managed with intravenous fluids. Later, gastrograffin study was normal and no surgical intervention was needed. Our patient also had radiation dermatitis Grade II which included moderate to brisk erythema, patchy moist desquamation mostly confined to skin folds and creases, and moderate edema.

\section{CONCLUSION}

Radiation enteritis can be managed conservatively at the start, but surgery is indicated when complications arise. Conservative treatment includes administration of topical anti-inflammatory agents, such as mesalazine or steroids (budesonide), glutamine, or the endoscopic application of formalin (in the large bowel) when there is bleeding. And finally, in case of chronic radiation enteropathy, surgery should be envisaged because of its progression to severe bowel necrosis, prolonged hospital stay, and the possibility of mortality.

\section{REFERENCES}

1. Gupta M, Dahiya J, Marwaha RK, Dureja H. Therapies in cancer treatment: An overview. Int J Pharm Pharm Sci 2015;7(4):1-9.

2. Stacey R, Green JT. Radiation-induced small bowel disease: Latest developments and clinical guidance. Ther Adv Chronic Dis 2014;5(1):15-29.

3. Green N, Iba G, Smith WR. Measures to minimize small intestine injury in the irradiated pelvis. Cancer 1975;35(6):1633-40.

4. Willett CG, Ooi CJ, Zietman AL, Menon V, Goldberg S, Sands BE, et al. Acute and late toxicity of patients with inflammatory bowel disease undergoing irradiation for abdominal and pelvic neoplasms. Int J Radiat Oncol Biol Phys 2000;46(4):995-8.

5. Bismar MM, Sinicrope FA. Radiation enteritis. Curr Gastroenterol Rep 2002;4:361-5

6. Hussain A, Mahmood H, Thomas A, Frazer C, El-Hasani S. Does chronic radiation enteritis pose a diagnostic challenge? A report of three cases. Hong Kong Med J 2008;14(4):327-30.

7. Romero Vázquez J, Caunedo Alvarez A, Rodríguez-Téllez M, Sánchez Yagüe A, Pellicer Bautista F, Herrerías Gutiérrez JM. Previously unknown stricture due to radiation therapy diagnosed by capsule endoscopy. Rev Esp Enferm Dig 2005;97(6):449-54.

8. Turina M, Mulhall AM, Mahid SS, Yashar C, Galandiuk S. Frequency and surgical management of chronic complications related to pelvic radiation. Arch Surg 2008;143(1):46-52. 